\title{
Dispersive regularizations and numerical discretizations for the inviscid Burgers equation
}

\author{
Georg A. Gottwald \\ School of Mathematics \& Statistics, University of Sydney, \\ NSW 2006, Australia. \\ gottwald@maths.usyd.edu.au
}

October 25, 2007

\begin{abstract}
We study centred second-order in time and space discretizations of the inviscid Burgers equation. Although this equation in its continuum formulation supports non-smooth shock wave solutions, the discrete equation generically supports smooth solitary wave solutions. Using backward error analysis we derive the modified equation associated with the numerical scheme. We identify three different equations, the Korteweg-de Vries (KdV) equation, the Camassa-Holm $(\mathrm{CH})$ equation and the $b=0$ member of the $b$-family. Solutions of the first two equations are solitary waves and do not converge to the shock solutions of the Burgers equation. The third equation however supports solutions which strongly approximate weak solutions of the Burgers equation. We corroborate our analytical results with numerical simulations.
\end{abstract}

PACS: 47.11.-j, 47.11.Bc, 47.35.Fg, 47.40.-x

MCS: 35Q51, 35Q53, 65M06, 37Kxx

Keywords: numerical dispersion, regularization, solitons, shock waves 


\section{Introduction}

The inviscid Burgers equation

$$
\frac{\partial u}{\partial t}+c \frac{\partial u}{\partial x}+\mu u \frac{\partial u}{\partial x}=0
$$

is the prototype equation for a shock-developing system [3, 13, 22]. An arbitrary smooth initial condition will develop a singularity of its gradient in finite time. The solution consists of one or more isolated shocks connected by smooth ramps. The equation involves linear and nonlinear advection. The nonlinear advection is responsible for the steepening. The strength of the nonlinearity is measured by the parameter $\mu$.

The occurrence of shock waves makes numerical integration of the Burgers equation particularly difficult. For the continuum Burgers equation (1) several attempts have been made to study so called regularized equations which support smooth regularized solutions. We distinguish here between viscously and dispersively regularization procedures. Whereas the viscously regularized Burgers equation

$$
u_{t}+c u_{x}+\mu u u_{x}=\nu u_{x x}
$$

supports solutions which in an $L_{1}$-sense converge strongly to weak solutions of the Burgers equation for $\nu \rightarrow 0$, conservative dispersively regularized equations such as the Korteweg-de Vries (KdV) equation [17]

$$
u_{t}+c u_{x}+\mu u u_{x}+\beta u_{x x x}=0,
$$

and the Camassa-Holm $(\mathrm{CH})$ equation [6]

$$
u_{t}+c u_{x}+\mu u u_{x}+\beta u_{x x x}-\alpha^{2} u_{x x t}+\frac{2}{3} \alpha^{2} \mu u_{x} u_{x x}+\frac{1}{3} \alpha^{2} \mu u u_{x x x}=0,
$$

support localized solitary waves. These dispersively regularized equations approximate the initial development of a smooth initial condition of the Burgers equation (1) until the solution develops into a weak shock solution. Then the approximation breaks down. The presence of linear dispersion in (3) and (4) balances the nonlinear steepening and gives rise to the formation of solitary waves. A recently introduced regularization, the $b=0$ member of the $b$-family [7]

$$
u_{t}+\mu u u_{x}-\alpha^{2} u_{x x t}-\alpha^{2} \mu u u_{x x x}=0
$$

does not contain linear dispersive terms. In contrast to the $\mathrm{KdV}$ equation and the CH-equation its solutions have been shown to strongly approximate weak solutions of the Burgers equation [2]. As supposed to viscously regularized equations such as (2) dispersively regularized equations such as (5) redistribute energy via nonlinear interactions rather than simply by dissipation. This has obvious advantages for long time integration where artificial energy dissipation will have a significant effect.

In the following we investigate finite-differencing methods to discretize (1) and study their solutions. We show that centred finite-difference equations of the Burgers equation lead to dispersive 
regularizations. The fact that discretization schemes of the Burgers equation can lead to dispersive regularizations had been previously studied [14] for semi-discretizations where time is kept continuous and only $x$ is discretized. Here we study fully discretized difference equations and determine under what conditions on the discretization the numerical scheme approximates the $\mathrm{KdV}$ equation, the $\mathrm{CH}$ equation or the $b=0$ member of the $b$-family. It turns out that solutions of symmetrically discretized numerical schemes generically do not consist of isolated nonsmooth shock waves but rather of smooth solitary waves, and only for special values of the discretization may approximate weak shock solutions. In Section 2 we present the numerical discretization under consideration. The resulting discretized equations are then analyzed in Section 3 by means of backward error analysis to derive the associated modified equations. In Sections $3.1-3.3$ we investigate the three cases when the modified equation is the $\mathrm{KdV}$ equation, the $\mathrm{CH}$ equation and the $b=0$ member of the $b$-family, respectively. We conclude with a discussion in Section 4 .

\section{Numerical discretization scheme}

The inviscid Burgers equation (1) is solved on a spatial box of length $L$ with $N$ equally spaced grid points $x_{j}=j \Delta x$ with grid spacing $\Delta x=L / N$. The temporal discretization is performed with a constant time-step $\Delta t$. We study here second-order in time and space centered finitedifferencing schemes $[11,19]$. In the following we introduce general discretization schemes for all terms of (1) which will allow for either explicit schemes or semi-implicit Crank-Nicolson schemes.

The temporal derivative is symmetrically discretized up to second order in $\Delta t$ as

$$
\left.\frac{\partial u}{\partial t}\right|_{t=n \Delta t, x=j \Delta x}=\frac{u_{j}^{n+a}-u_{j}^{n-a}}{2 a \Delta t} .
$$

Superscripts denote temporal discretization and subscripts denote spatial discretization. One could have also included symmetric spatial averaging in (6), but we find that this is not necessary for our purposes. The linear advection term is discretized by means of a centred spatial differencing and centered temporal averaging to assure second order accuracy in $\Delta x$ and $\Delta t$

$$
\left.c \frac{\partial u}{\partial x}\right|_{t=n \Delta t, x=j \Delta x}=\frac{c}{2}\left(\frac{u_{j+s}^{n+b}-u_{j-s}^{n+b}}{2 s \Delta x}+\frac{u_{j+s}^{n-b}-u_{j-s}^{n-b}}{2 s \Delta x}\right) .
$$

Note that the prefactor $1 / 2$ has to be omitted when the discretization does not involve temporal averaging for $b=0$. The nonlinear advection term is discretized similarly up to second-order in space and time according to

$\left.\mu u \frac{\partial u}{\partial x}\right|_{t=n \Delta t, x=j \Delta x}=\frac{1}{2} \mu\left(\frac{1}{2}\left(u_{j+y}^{n+d}+u_{j-y}^{n+d}\right) \frac{u_{j+z}^{n+d}-u_{j-z}^{n+d}}{2 z \Delta x}+\frac{1}{2}\left(u_{j+y}^{n-d}+u_{j-y}^{n-d}\right) \frac{u_{j+z}^{n-d}-u_{j-z}^{n-d}}{2 z \Delta x}\right)$.

Again the two prefactors of $1 / 2$ occur only for $d=0$ and $y=0$, respectively. The Burgers equation (1) discretized using (6)-(8) can be solved for $u_{j}^{n+a}$ either explicitly or by matrix inversion in the case that the choice of $a, b$ and $d$ imply an implicit scheme. 


\section{Backward error analysis and the modified equation}

The idea of backward error analysis (see for example [11]) is that a numerical scheme for (1) such as the scheme (6)-(8) is not solving the original equation - in our case the Burgers equation (1) - but rather approximates up to a certain order in $\Delta x$ and $\Delta t$ a so called modified equation. To derive the modified equation for the above scheme we use Taylor-expansions of $u_{j}^{n}$. In particular, we evaluate

$$
u_{j \pm k}=u_{j} \pm k \Delta x\left(u_{x}\right)_{j}+\frac{1}{2} k^{2} \Delta x^{2}\left(u_{x x}\right)_{j} \pm \frac{1}{6} k^{3} \Delta x^{3}\left(u_{x x x}\right)_{j}+\mathcal{O}\left(\Delta x^{4}\right),
$$

where $u$ is evaluated at a fixed point in time, let's say at $t=n \Delta t$, and analogously

$$
u^{n \pm l}=u^{n} \pm l \Delta t\left(u_{t}\right)^{n}+\frac{1}{2} l^{2} \Delta t^{2}\left(u_{t t}\right)^{n} \pm \frac{1}{6} l^{3} \Delta t^{3}\left(u_{t t t}\right)^{n}+\mathcal{O}\left(\Delta t^{4}\right)
$$

where $u$ is evaluated at a fixed spatial grid point, let's say at $x_{j}=j \Delta x$. We consider here discretizations $\mathcal{O}(\Delta x) \sim \mathcal{O}(\Delta t)$ and also restrict ourselves in the following to small amplitude waves with $\mathcal{O}(\mu) \sim \mathcal{O}(\Delta x)$. Using the Taylor expansions (9) and (10) for $u_{j}^{n}$ we can rewrite the individual discretizations (6)-(8) in terms of the continuous spatial and temporal derivatives of $u$. First (6) is expanded as

$$
\left.\frac{\partial u}{\partial t}\right|_{t=n \Delta t, x=j \Delta x}=u_{t}+\frac{1}{6} a^{2} \Delta t^{2} u_{t t t}+\mathcal{O}\left(\Delta t^{4}\right),
$$

where here and in the following the right hand side is evaluated at time $t=n \Delta t$ and spatial location $x=j \Delta x$. Similarly (7) becomes

$$
\left.\frac{\partial u}{\partial x}\right|_{t=n \Delta t, x=j \Delta x}=u_{x}+\frac{1}{6} s^{2} \Delta x^{2} u_{x x x}+\frac{1}{2} b^{2} \Delta t^{2} u_{t t x}+\mathcal{O}\left(\Delta x^{4}, \Delta t^{4}\right),
$$

and (8)

$$
\begin{gathered}
\left.\mu u \frac{\partial u}{\partial x}\right|_{t=n \Delta t, x=j \Delta x}=\mu u u_{x}+\mu\left(\frac{1}{6} z^{2} \Delta x^{2}+\frac{1}{2} c^{2} d^{2} \Delta t^{2}\right) u u_{x x x}+\mu\left(\frac{1}{2} y^{2} \Delta x^{2}+\frac{3}{2} c^{2} d^{2} \Delta t^{2}\right) u_{x} u_{x x} \\
+\mathcal{O}\left(\Delta x^{4}, \Delta t^{4}, \Delta x^{2} \Delta t^{2}, \mu^{2} \Delta x^{2}, \mu^{2} \Delta t^{2}\right)
\end{gathered}
$$

To obtain the last equation we substituted the zeroth order equation $u_{t}=-c u_{x}$ back into the equation. We summarize and write down the modified equation for our discretization (6)-(8) as

$$
\begin{aligned}
u_{t}+c u_{x}+\mu u u_{x}= & -\frac{1}{6} a^{2} \Delta t^{2} u_{t t t}-\frac{1}{2} c b^{2} \Delta t^{2} u_{t t x}-\frac{1}{6} c s^{2} \Delta x^{2} u_{x x x} \\
& -\mu\left(\frac{1}{2} y^{2} \Delta x^{2}+\frac{3}{2} c^{2} d^{2} \Delta t^{2}\right) u_{x} u_{x x}-\mu\left(\frac{1}{6} z^{2} \Delta x^{2}+\frac{1}{2} c^{2} d^{2} \Delta t^{2}\right) u u_{x x x} \\
& +\mathcal{O}\left(\Delta x^{4}, \Delta t^{4}, \Delta x^{2} \Delta t^{2}, \mu^{2} \Delta x^{2}, \mu^{2} \Delta t^{2}\right)
\end{aligned}
$$

This modified equation differs from the original Burgers equation by additional linear and nonlinear dispersive terms. The nonlinear dispersive terms are of the same form as the nonlinear dispersive terms of the Camassa-Holm equation (4). The exclusive occurrence of dispersive terms is due to our scheme being centred in time and space. If we had used non-centred methods in time or space we would have obtained either damping or amplifying terms depending 
on the scheme. We may express time derivatives as space derivatives using the lowest order balance $u_{t}+c u_{x}=\mathcal{O}\left(\mu, \Delta x^{2}, \Delta t^{2}\right)$, to realize that at $\mathcal{O}\left(\mu, \Delta x^{2}, \Delta t^{2}\right)$ the modified equation is the KdV equation (3). In the following we will investigate the influence of the higher order terms.

Inspection of the modified equation (14) suggests that we may expect to obtain the three dispersively regularized equations of interest for us as modified equations at $\mathcal{O}\left(\Delta x^{4}, \Delta t^{4}, \Delta x^{2} \Delta t^{2}\right.$, $\mu^{2} \Delta x^{2}, \mu^{2} \Delta t^{2}$ ), namely the Korteweg-de Vries (KdV) equation (3), the Camassa-Holm (CH) equation (4) and the $b=0$ member of the $b$-family (5). In the following we show that for specific discretizations, i.e. appropriate values of $a, b, d, y, z$ and $\Delta x$ and $\Delta t$, one may obtain discretizations whose associated modified equations coincide with each of these three dispersively regularizations of the inviscid Burgers equation (1).

In order to do so we need to substitute the various temporal derivatives occurring in the modified equation (14), in particular the terms $u_{t t t}$ and $u_{t t x}$. For example, the KdV equation (3) does not contain any term of the form $u_{x x t}$, whereas the $\mathrm{CH}$ equation (4) and the nonlinearly regularized Burgers equation (5) do. We partially substitute occurring time derivatives by the first order approximation $u_{t}=-c u_{x}-\mu u u_{x}$. We introduce a free parameter $q$ which controls the splitting of the temporal derivatives. We write

$$
u_{t t t}=\begin{array}{r}
q\left(-c^{3} u_{x x x}-3 \mu c^{2}\left(3 u_{x} u_{x x}+u u_{x x x}\right)\right) \\
(1-q)\left(c^{2} u_{x x t}-2 \mu c^{2}\left(3 u_{x} u_{x x}+u u_{x x x}\right)\right) .
\end{array}
$$

The remaining temporal derivative $u_{t t x}$ in (14) can be fully substituted as

$$
u_{t t x}=c^{2} u_{x x x}+2 \mu c\left(3 u_{x} u_{x x}+u u_{x x x}\right) .
$$

It turns out that the free parameter $q$ is sufficient and one does not need to introduce further free parameters to only allow for partial substitutions of temporal derivatives in $u_{t t x}$ and $u_{x x x}$. Inserting (15) and (16) into (14) we obtain

$$
u_{t}+c u_{x}+\mu u u_{x}+C\left(u_{x x x}\right) u_{x x x}+C\left(u_{x x t}\right) u_{x x t}+C\left(u_{x} u_{x x}\right) u_{x} u_{x x}+C\left(u u_{x x x}\right) u u_{x x x}=0,
$$

with

$$
\begin{aligned}
C\left(u_{x x x}\right)= & \frac{1}{6} s^{2} c \Delta x^{2}-\frac{1}{6} q a^{2} c^{3} \Delta t^{2}+\frac{1}{2} b^{2} c^{3} \Delta t^{2} \\
C\left(u_{x x t}\right)= & \frac{1}{6}(1-q) a^{2} c^{2} \Delta t^{2} \\
= & \frac{1}{c} C\left(u_{x x x}\right)-\frac{1}{6} s^{2} \Delta x^{2}+\frac{1}{6} a^{2} c^{2} \Delta t^{2}-\frac{1}{2} b^{2} c^{2} \Delta t^{2} \\
C\left(u_{x} u_{x x}\right)= & -\frac{1}{2} \mu(2+q) a^{2} c^{2} \Delta t^{2}+3 \mu b^{2} c^{2} \Delta t^{2}+\frac{3}{2} \mu d^{2} c^{2} \Delta t^{2} \\
& +\frac{1}{2} \mu y^{2} \Delta x^{2} \\
C\left(u u_{x x x}\right)= & -\frac{1}{6} \mu(2+q) a^{2} c^{2} \Delta t^{2}+\mu b^{2} c^{2} \Delta t^{2}+\frac{1}{2} \mu d^{2} c^{2} \Delta t^{2} \\
& +\frac{1}{6} \mu z^{2} \Delta x^{2} \\
= & \frac{1}{3} C\left(u_{x} u_{x x}\right)+\frac{1}{6} \mu\left(z^{2}-y^{2}\right) \Delta x^{2} .
\end{aligned}
$$

It is this form of the modified equation we will be working with from now on. 


\subsection{Korteweg-de Vries equation}

In this Section we show that one can choose the parameters of the discretization of (6)-(8) in such a way that its associated modified equation (17) is the famous KdV equation (3) which we recall here

$$
u_{t}+c u_{x}+\mu u u_{x}+\beta u_{x x x}=0 .
$$

Inspection of (17) reveals that any numerical scheme and its dispersive regularization approximates the $\mathrm{KdV}$ equation up to $\mathcal{O}\left(\mu \Delta x^{2}, \mu \Delta t^{2}\right)$, and effectively simulates the $\mathrm{KdV}$ equation rather than the Burgers equation (1). Our aim here is to determine the conditions for a numerical scheme that approximates the $\mathrm{KdV}$ equation up to $\mathcal{O}\left(\Delta x^{4}, \Delta t^{4}, \mu^{2} \Delta x^{2}, \mu^{2} \Delta t^{2}\right)$. To obtain (3) as the modified equation up to this order we need to choose our discretization scheme and our free parameter in order to assure

$$
\begin{aligned}
C\left(u_{x x t}\right) & =0 \\
C\left(u_{x} u_{x x}\right) & =0 \\
C\left(u u_{x x x}\right) & =0 .
\end{aligned}
$$

These conditions translate to

$$
\begin{aligned}
q & =1 \\
-(2+q) a^{2} A^{2}+6 b^{2} A^{2}+3 d^{2} A^{2}+y^{2} & =0 \\
y & =z,
\end{aligned}
$$

where we introduced

$$
A^{2}=c^{2} \frac{\Delta t^{2}}{\Delta x^{2}} .
$$

Note that $A^{2} \leq 1$ is required for numerical stability as a Courant-Friedrichs-Levy condition. This assures that the numerical scheme with discretization $\Delta x$ and $\Delta t$ is able to resolve processes which propagate with the physical wave speed $c$. Condition (23) yields

$$
A^{2}=\frac{1}{3} \frac{y}{a^{2}-2 b^{2}-d^{2}} \leq 1
$$

Any numerical scheme which satisfies $0<A^{2} \leq 1$ with $A^{2}$ given by (26) together with $y=z$ has the $\mathrm{KdV}$ equation with linear dispersion

$$
\beta=c \frac{\Delta x^{2}}{6}\left(s^{2}-a^{2} A^{2}+3 b^{2} A^{2}\right) .
$$

as its modified equation up to $\mathcal{O}\left(\Delta x^{4}, \Delta t^{4}, \mu^{2} \Delta x^{2}, \mu^{2} \Delta t^{2}\right)$. For example, for a given numerical scheme with given $\Delta x$ and $\Delta t$ that satisfies (24) and the inequality (26), one may choose the linear advective speed $c$ such that $A^{2}=c^{2} \Delta t^{2} / \Delta x^{2}$. One may similarly prescribe $c$ and $\Delta x$ $(\Delta t)$, which then subsequently fixes $\Delta t(\Delta x)$ to satisfy condition (26).

The Korteweg-de Vries equation is integrable, supports smooth solutions, and its initial value problem is solvable via the inverse scattering transformation [1]. In particular, equation (3) supports a one-parameter family of soliton solutions of the form

$$
u\left(x-c_{\mathrm{sol}} t\right)=a \operatorname{sech}^{2}\left(w\left(x-c_{\mathrm{sol}} t\right)\right),
$$


with amplitude

$$
a=12 \frac{\beta}{\mu} w^{2}
$$

and speed

$$
c_{\mathrm{sol}}=c+4 \beta w^{2}
$$

The existence of solitons crucially depends on the balance of the nonlinear steepening provided by $u u_{x}$ and the linear dispersion $u_{x x x}$. The linear dispersion can be attributed entirely to the numerical scheme with $\beta=\beta\left(\Delta x^{2}\right)$. Hence these solitary waves are spurious in the sense that the continuum system (1) does not support them and they are purely an artefact of the chosen numerical integration scheme.

Note that the constant advection with $c \neq 0$ is crucial for the existence of the linear dispersive term $C\left(u_{x x x}\right)$ of the modified equation. If we initially had transformed into the system moving with the constant advection velocity $c$ by introducing $\xi=x-c t$ to eliminate the $c u_{x}$ term, there would be no linear dispersive terms in the modified equation (17).

To illustrate the effect of the discretization and the modified equation we look at a particular example. We choose $a=2, b=1, d=0$ and $z=y=s=1$, which makes the numerical scheme (6)-(8) explicit. Condition (23) is satisfied with $0<A^{2}=1 / \sqrt{6}<1$ assuring a stable scheme. This choice of parameters implies for the linear dispersion coefficient $\beta=5 c \Delta x^{2} / 36$. In Fig. 1 we show results from a numerical simulation of the scheme (6)-(8) for the Burgers equation for periodic boundary conditions. The results clearly show that the KdV equation is the modified equation and that the solitary wave (27) is the appropriate solution.
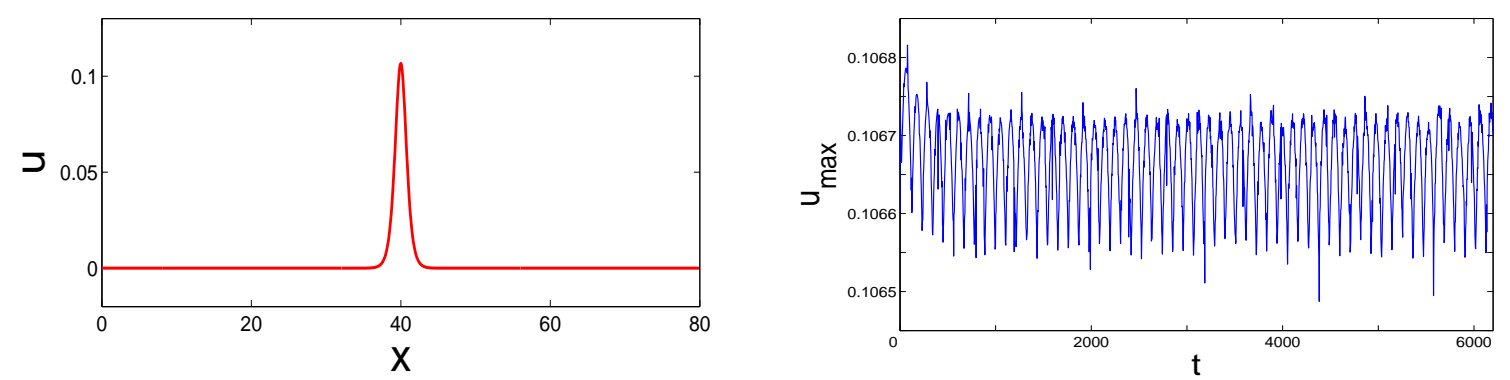

Figure 1: Left: Plot of the solitary wave (27) for the numerical scheme for the Burgers equation (6)-(8) with $a=2, b=1, d=0, z=y=s=1$ corresponding to the KdV equation. We use $\Delta x=0.08$ for the spatial discretization on a ring with $L=80$. Equation parameters are $\mu=0.1$ and $c=1$ which implies $\Delta t=0.0327$. Right: Plot of the maximal amplitude $u_{\max }$ of the solitary wave as a function of time. The solitary wave solution retains amplitude and shape.

To illustrate this further we look at the two-soliton solution of (3) parameterized by $w_{1}$ and $w_{2}$ 
$[21,16]$

$$
\begin{aligned}
u(x, t)=12 \frac{\beta}{\mu} & \left(\frac{w_{1}^{2}-w_{2}^{2}}{\left(w_{1} \operatorname{coth}\left(w_{1}\left(x-\frac{L}{2}\right)\right)-w_{2} \tanh \left(w_{2}\left(x-\frac{L}{2}\right)\right)\right)^{2}}\right) \\
& \times\left(\frac{w_{1}^{2}}{\sinh ^{2}\left(w_{1}\left(x-\frac{L}{2}\right)\right)}+\frac{w_{2}^{2}}{\cosh ^{2}\left(w_{2}\left(x-\frac{L}{2}\right)\right)}\right) .
\end{aligned}
$$

In a periodic box, the two solitary waves elastically interact. In Fig. 2 we show the maximal amplitude which is constant for the times when the two solitons are well separated and drops when they interact.

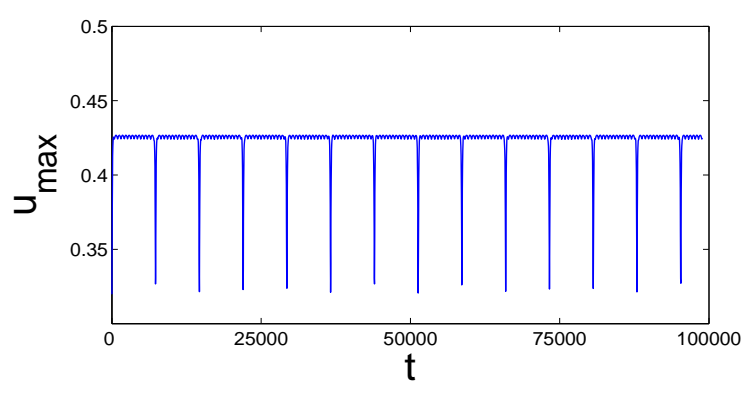

Figure 2: Plot of the maximal amplitude as a function of time for the two-soliton solution (30) of the KdV equation. We use the same numerical scheme and the same parameters as in Fig. 1.

\subsection{Camassa-Holm equation}

In [6] the Camassa-Holm equation (4) which we recall as

$$
u_{t}+c u_{x}+\mu u u_{x}+\beta u_{x x x}-\alpha^{2} u_{x x t}+\frac{2}{3} \alpha^{2} \mu u_{x} u_{x x}+\frac{1}{3} \alpha^{2} \mu u u_{x x x}=0,
$$

was introduced to describe shallow water waves. It has been derived in an asymptotic multiscale expansion for water waves $[8,9]$. The order of the asymptotic approximation is one order better compared to the $\mathrm{KdV}$ equation. As with its lower order cousin the CH-equation is integrable [8].

In $[9,20]$ solutions of (4) were investigated. The CH-equation (4) supports solitary waves, periodic waves, peakons for which the first derivative is discontinuous, and cuspons for which the first derivative has a singularity.

We first focus on the one-parameter family of solitary travelling waves - parametrized by the wave speed $c_{\text {sol }}$ - which is given by

$$
u(\tau)=3 \frac{\delta}{\mu} \operatorname{sech}^{2} \frac{\sqrt{\delta}}{2} \tau,
$$

where $\delta=c_{\mathrm{sol}}-c$. The spatial dependence of the solitary wave solution is parametrized by the variable $\tau$. The relation between $\tau$ and $x$ is given by the Sundman transformation

$$
\frac{d x}{d \tau}=\sqrt{\beta+\alpha^{2} c_{\mathrm{sol}}-\alpha^{2} \delta \operatorname{sech}^{2} \frac{\sqrt{\delta}}{2} \tau},
$$


which can be explicitly solved to yield

$$
\begin{aligned}
x(\tau)= & 2 \sqrt{\frac{\beta+\alpha^{2} c_{\mathrm{sol}}}{\delta}} \sinh ^{-1}\left(\sqrt{\frac{\beta+\alpha^{2} c_{\mathrm{sol}}}{\beta+\alpha^{2} c}} \sinh \frac{\sqrt{\delta}}{2} \tau\right) \\
& -2 \sqrt{\alpha^{2}} \tanh ^{-1}\left(\sqrt{\alpha^{2} \delta} \frac{\tanh \frac{\sqrt{\delta}}{2} \tau}{\sqrt{\alpha^{2} \delta \tanh ^{2} \frac{\sqrt{\delta}}{2} \tau+\beta+\alpha^{2} c}}\right) .
\end{aligned}
$$

For the regularizing case $\alpha^{2}>0$ the conditions for a travelling wave are [9]

$$
\begin{aligned}
\delta & >0 \\
\beta+\alpha^{2} c & >0 .
\end{aligned}
$$

In this section we write down the conditions such that the numerical scheme (6)-(8) contains the CH-equation (4) as its modified equation (17). Using scaling transformations of $u, x$ and $t$, the coefficients of the CH-equation may be changed. However, in [10] it was shown that the following conditions for its coefficients are essential for integrability of the $\mathrm{CH}$ equation (4)

$$
\begin{aligned}
C\left(u_{x} u_{x x}\right): C\left(u u_{x x x}\right) & =2: 1 \\
C\left(u_{x x t}\right) C\left(u u_{x}\right): C\left(u u_{x x x}\right) C\left(u_{t}\right) & =3: 1 .
\end{aligned}
$$

Moreover, we require $\alpha^{2}$ to be positive. Identifying $\alpha^{2}=-C\left(u_{x x t}\right)$ this amounts to

$$
C\left(u_{x x t}\right)<0 \text {. }
$$

Using the definitions for the coefficients (18)-(21) the conditions (36)-(38) can be written as

$$
\begin{array}{r}
-(2+q) a^{2} A^{2}+6 b^{2} A^{2}+3 d^{2} A^{2}=2 z^{2}-3 y^{2} \\
(7+2 q) a^{2} A^{2}-18 b^{2} A^{2}-9 d^{2} A^{2}=3 z^{2} \\
1-q<0
\end{array}
$$

where again $A^{2}$ is defined by $(25)$ and is subject to the Courant-Friedrichs-Levy stability condition

$$
0<A^{2} \leq 1
$$

Note that we have the additional restriction $c_{\mathrm{sol}} \Delta t / \Delta x \leq 1$ to assure that the solitary wave propagation can be resolved by the numerical scheme. Combining (39) and (40) we obtain

$$
3 a^{2} A^{2}-6 b^{2} A^{2}-3 d^{2} A^{2}=7 z^{2}-6 y^{2} .
$$

Combining conditions (39) and (41) we obtain

$$
3 a^{2} A^{2}-6 b^{2} A^{2}-3 d^{2} A^{2}+2 z^{2}-3 y^{2}<0,
$$

which together with (43) implies

$$
z<y \text {. }
$$


Rewriting (43) we find

$$
A^{2}=\frac{1}{3} \frac{7 z^{2}-6 y^{2}}{a^{2}-2 b^{2}-d^{2}}
$$

which further constraints the admissible parameters for the discretization because $0<A^{2} \leq$ 1 is required as the Courant-Friedrichs-Levy condition. For each admissible combination of discretization parameters we may calculate the free parameter $q$ from (40) as

$$
q=-\frac{1}{2 a^{2} A^{2}}\left(7 a^{2} A^{2}-18 b^{2} A^{2}-9 d^{2} A^{2}-3 z^{2}\right),
$$

with the additional constraint (41) requiring that $q>1$. This defines the numerical scheme for the modified equation to be the $\mathrm{CH}$ equation (4).

If we are additionally interested in the solitary wave solution (31) we need to further satisfy conditions (34) and (35). Identifying $\beta=C\left(u_{x x x}\right)$ conditions (34) and (35) can be written as

$$
\begin{aligned}
c_{\text {sol }}-c & >0 \\
s^{2}-a^{2} A^{2}+3 b^{2} A^{2} & >0 .
\end{aligned}
$$

If the conditions for the validity of the $\mathrm{CH}$ equation (44) - (46) are satisfied we expect the numerical scheme (6)-(8) to model the $\mathrm{CH}$ equation up to $\mathcal{O}\left(\Delta x^{4}, \Delta t^{4}, \mu^{2} \Delta x^{2}, \mu^{2} \Delta t^{2}\right)$. If additionally the conditions for the existence of travelling waves for the $\mathrm{CH}$ equation (47) and (48) are satisfied, we expect the numerical scheme (6)-(8) to support the solitary wave (31). For example, $a=3, b=3, d=0, z=1, y=2$ and $s=1$ is such a choice of parameters and implies $q=98 / 17$ and $A^{2}=17 / 27<1$. Because $a=b=3$ we may write the scheme as a one-step scheme. For these parameters the numerical scheme (6)-(8) is semi-implicit, which is unconditionally stable [19]. We solve the system of equations numerically using LU-decomposition.

In Fig. 3 we show results from a numerical simulation of the scheme (6)-(8) for the CH equation, again for periodic boundary conditions. As for the KdV equation we see clearly that the modified equation is the $\mathrm{CH}$ equation and the numerical scheme preserves the solitary wave solution (31).

In the remainder of this Section we investigate peakon solutions of the $\mathrm{CH}$ equation. Condition (35), which we may rewrite as $C\left(u_{x x x}\right)>c C\left(u_{x x t}\right)$, restricts the solutions of the $\mathrm{CH}$ equation (4) to the smooth solitary wave solution (31). For $C\left(u_{x x x}\right)=c C\left(u_{x x t}\right)$ however, so called peakons are supported for which the first derivative is not continuous. The $\mathrm{CH}$ equation in the peakon limit reads as

$$
u_{t}+c u_{x}+\mu u u_{x}-\alpha^{2} u_{x x t}-c \alpha^{2} u_{x x x}+\frac{2}{3} \alpha^{2} \mu u_{x} u_{x x}+\frac{1}{3} \alpha^{2} \mu u u_{x x x}=0,
$$

which we have cast here in the form where we moved into a frame of reference moving with speed $-c$. This equation only involves nonlinear dispersion, and supports a one-parameter family of solutions $u=c \exp (|x-c t|)$. The numerical scheme (6)-(8) have the peakon equation (49) as its modified equation for the choice of parameters $a=7, b=4, d=2, s=1, y=16$ and $z=15$ implying $A^{2}=1$. However, we found that this explicit scheme is numerically unstable. 

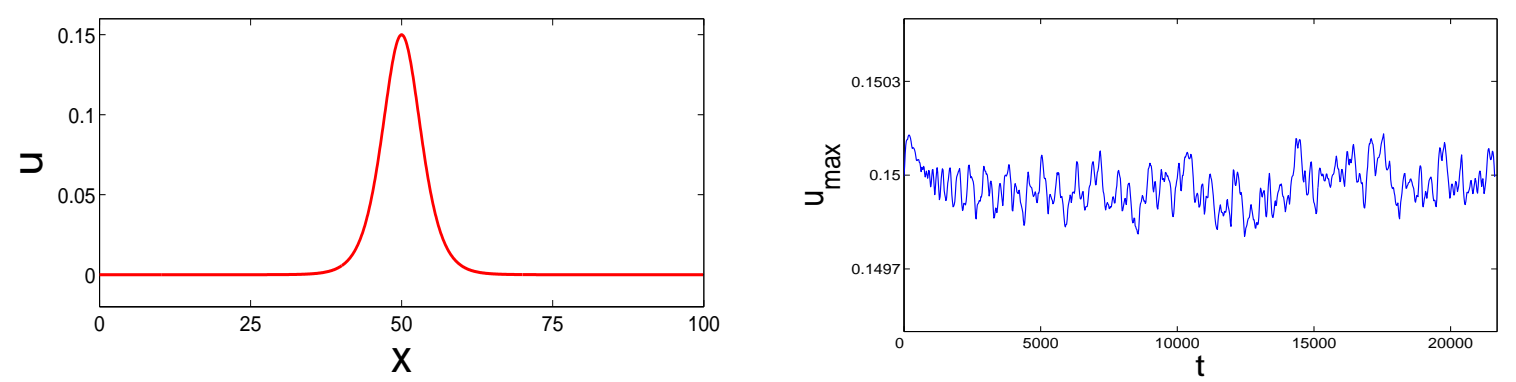

Figure 3: Left: Plot of the solitary wave (31) for the numerical scheme for the Burgers equation (6)-(8) with $a=3, b=3, d=0, z=1, y=2, s=1$ and $q=98 / 17$ corresponding to the $\mathrm{CH}$ equation. We choose $\Delta x=0.1$. The equation parameters are $\mu=0.1$ and $c=1.1$ which implies $\Delta t=0.0721$. Right: Plot of the maximal amplitude $u_{\max }$ of the solitary wave as a function of time.

\subsection{Regularized Burgers equation}

Whereas the $\mathrm{KdV}$ equation and the $\mathrm{CH}$ equation are regularizing the Burgers equation by their inherent linear dispersion, the $b=0$ member of the $b$-family (5) which we recall

$$
u_{t}+\mu u u_{x}-\alpha^{2} u_{x x t}-\alpha^{2} \mu u u_{x x x}=0,
$$

does not contain any linear dispersive terms [7]. In [2] it was shown that solutions of this equation strongly converge to weak solutions of the Burgers equation. The particular mechanism of regularization can be seen best if we write (5) as

$$
v_{t}+u v_{x}=0 \quad \text { where } \quad u=\left(1-\alpha^{2} \partial_{x x}\right)^{-1} v .
$$

This is analogous to the regularization used in $\alpha$-Euler models $[15,4,5]$ in which the advecting velocity is smoothed with respect to the advected velocity. As in $\alpha$-Euler models the smoothing is performed by an inverse Helmholtz operator.

To allow for the asymptotic resubstitutions of temporal derivatives (15) and (16) which were used to obtain (18)-(21) we move in a frame of reference moving with $-c$ and write (5) as

$$
u_{t}+c u_{x}+\mu u u_{x}-\alpha^{2} u_{x x t}-c \alpha^{2} u_{x x x}-\alpha^{2} \mu u u_{x x x}=0 .
$$

We now ask the same question as before for the $\mathrm{KdV}$ equation or the $\mathrm{CH}$ equation. Can we find a numerical discretization, i.e. parameters for the temporal discretization $a, b, d$ and for the spatial discretization $s, y$ and $z$, and a free parameter $q$ such that (51) is the modified equation for (6)-(8). In particular, we require

$$
\begin{aligned}
C\left(u_{x} u_{x x}\right) & =0 \\
C\left(u u_{x x x}\right) & =\mu C\left(u_{x x t}\right)<0 \\
C\left(u_{x x x}\right) & =c C\left(u_{x x t}\right) .
\end{aligned}
$$

From condition (54), which we have already encountered for the peakon equation (49), we deduce that

$$
s^{2}-a^{2} A^{2}+3 b^{2} A^{2}=0,
$$


where $A^{2}$ is again defined via (25). Using (18)-(21) condition (52) implies

$$
C\left(u u_{x x x}\right)=\frac{1}{6} \mu\left(z^{2}-y^{2}\right) \Delta x^{2} .
$$

Since according to condition $(53)$ we have $C\left(u u_{x x x}\right)<0$ we need to impose

$$
y>z
$$

Condition (52) reads as

$$
-(2+q) a^{2} A^{2}+6 b^{2} A^{2}+3 d^{2} A^{2}+y^{2}=0 .
$$

Condition (53) together with (56) reads as

$$
z^{2}-y^{2}-s^{2}+q a^{2} A^{2}-3 b^{2} A^{2}=0,
$$

which together with (58) results in

$$
-s^{2}-2 a^{2} A^{2}+3 b^{2} A^{2}+3 d^{2} A^{2}+z^{2}=0 .
$$

Combining (55) and (60) we obtain the condition for our discretization parameters by eliminating $A^{2}$ as

$$
\left(s^{2}-\frac{1}{3} z^{2}\right) a^{2}+\left(z^{2}-2 s^{2}\right) b^{2}-s^{2} d^{2}=0,
$$

and may also determine

$$
A^{2}=\frac{1}{3} \frac{z^{2}-3 s^{2}}{b^{2}-d^{2}} \leq 1
$$

Given a solution of (61) satisfying the stability constraint (62) the free parameter for the resubstitution of the temporal derivatives can be calculated directly from, for example, (59) as

$$
q=\frac{1}{a^{2} A^{2}}\left(s^{2}+3 b^{2} A^{2}+y^{2}-z^{2}\right) .
$$

One possible choice of parameters satisfying (61) and (62) is

$$
a=2, \quad b=0, \quad d=1, \quad s=2, \quad z=3, \quad y=4,
$$

which implies $A^{2}=1$. In Fig. 4 we show results from a numerical realization of this discretization scheme. Due to the spatial discretization $s \neq z \neq y$ there are three subgrids which evolve independently. We therefore perform after every 20 time steps a spatial averaging according to $u_{j}=\left(u_{j-1}+2 u_{j}+u_{j+1}\right) / 4$. Note that this introduces some small artificial viscosity to the scheme. Therefore the actual numerical scheme associated with this spatial averaging deviates from the discretization scheme (6)-(8). Hence the modified equation deviates slightly from the $b=0$ member of the $b$-family (51). However, if the same amount of spatial averaging is applied to discretization schemes whose associated modified equation are the $\mathrm{KdV}$ equation or the $\mathrm{CH}$ equation, no weak shock solutions develop but the dynamics is still dominated by (now damped) solitary waves and their near elastic interaction. In those cases the damping associated with the spatial averaging is simply a small perturbation to the otherwise integrable nonlinear wave equations which support smooth solitary wave solutions. Hence the introduced viscosity does not simply transform the modified equation into the viscously regularized Burgers equation (2), 
but rather stabilizes the dispersively regularized equation (51). We have further checked this by letting the parameters of the numerical scheme associated with (51) vary slightly. For example, for $\Delta t \rightarrow 0.9 \Delta t^{\star}$ (for fixed $\Delta x$ ) where $\Delta t^{\star}$ is the temporal spacing consistent with (52)-(54), one observes solitary waves emanating from the initial pulse condition rather than weak shock solutions as depicted in Fig. 4. This is consistent with our analytical findings of Section 3.1.

As can be seen from (56) one can control the value of $\alpha^{2}$ by increasing $y$. For example if all parameters are kept as in Fig. 4 a value of $y=5$ leads to $\alpha^{2} \approx 1.6 \Delta x$. However, one does not observe a sharper and sharper shock front for smaller values of the smoothing length $\alpha^{2}$ as expected from the theory [2]. We have tested the shape of $u$ for different values of $\alpha^{2}$ and found no difference. This suggests that the viscosity associated with the averaging is the important dynamical mechanism to produce regularized shocks, and that the numerical discretization associated with (51) only assures that no linear dispersion is present. However, one may achieve better approximation to a shock wave by reducing the discretization. In Fig. 4 we show results for two spatial and temporal discretizations. We chose $\Delta x=0.025$ which then implies $\Delta t=\Delta x=0.025$, and for the lower right panel we use half the grid size $\Delta x=0.0125$ which implies $\Delta t=0.0125$. These two discretizations correspond to $\alpha^{2}=-C\left(u_{x x t}\right)=0.00073$ and $\alpha^{2}=0.00018$, respectively. However, as discussed above, the better approximation of a shock wave for $\alpha^{2}=0.00018$ is not due to $\alpha^{2}$ being smaller but simply due to the finer discretization applied.

Whereas the parameter $\alpha^{2}$ usually is motivated on physical grounds as a length scale over which fluctuations are averaged out (see for example [15]), the parameter $\alpha^{2}$ arises here solely through the numerical discretization scheme chosen. For example, for the parameters chosen to obtain Fig. 4 we obtain $\alpha^{2}=0.00073 \approx \Delta x^{2}$ for the coarse grid and $\alpha^{2}=0.00018 \approx(\Delta x / 2)^{2}$ for the finer grid. Hence the parameter $\alpha^{2}$ cannot be interpreted solely as a length scale over which smoothing takes place since in the finer grid there are no grid points on that scale.

The link between regularization procedures such as Helmholtz regularization and numerical schemes had been studied before, for example in [12]. However here we achieve an $\alpha$-regularization with an explicit scheme.

\section{Summary}

We have investigated different numerical schemes to discretize the Burgers equation (1). Usually either artificial viscosity is added or upwinding methods are employed to control the problems arising with the steep gradients $[11,19]$. In contrast, we have focused here on dispersive regularizations. In particular we analyzed second-order discretizations which are symmetric in time and space for small amplitude solutions. We analyzed the numerical discretizations of the Burgers equation by means of backward error analysis and derived the respective associated modified equations up to $\mathcal{O}\left(\Delta x^{4}, \Delta t^{4}, \mu^{2} \Delta x^{2}, \mu^{2} \Delta t^{2}\right)$.

The discretization generically generates the Korteweg-de Vries equations as its corresponding modified equation at $\mathcal{O}\left(\mu \Delta x^{2}, \mu \Delta t^{2}\right)$. In a numerical simulation this causes an arbitrary smooth initial condition to evolve into a train of solitary waves which then subsequently interact (up to this asymptotic order) elastically in a periodic domain; the additional dispersive linear and nonlinear terms in (17) are a small perturbation of the integrable Korteweg-de Vries equation. We illustrate this in Fig. 5 where we show the temporal evolution of an initial bump. We 

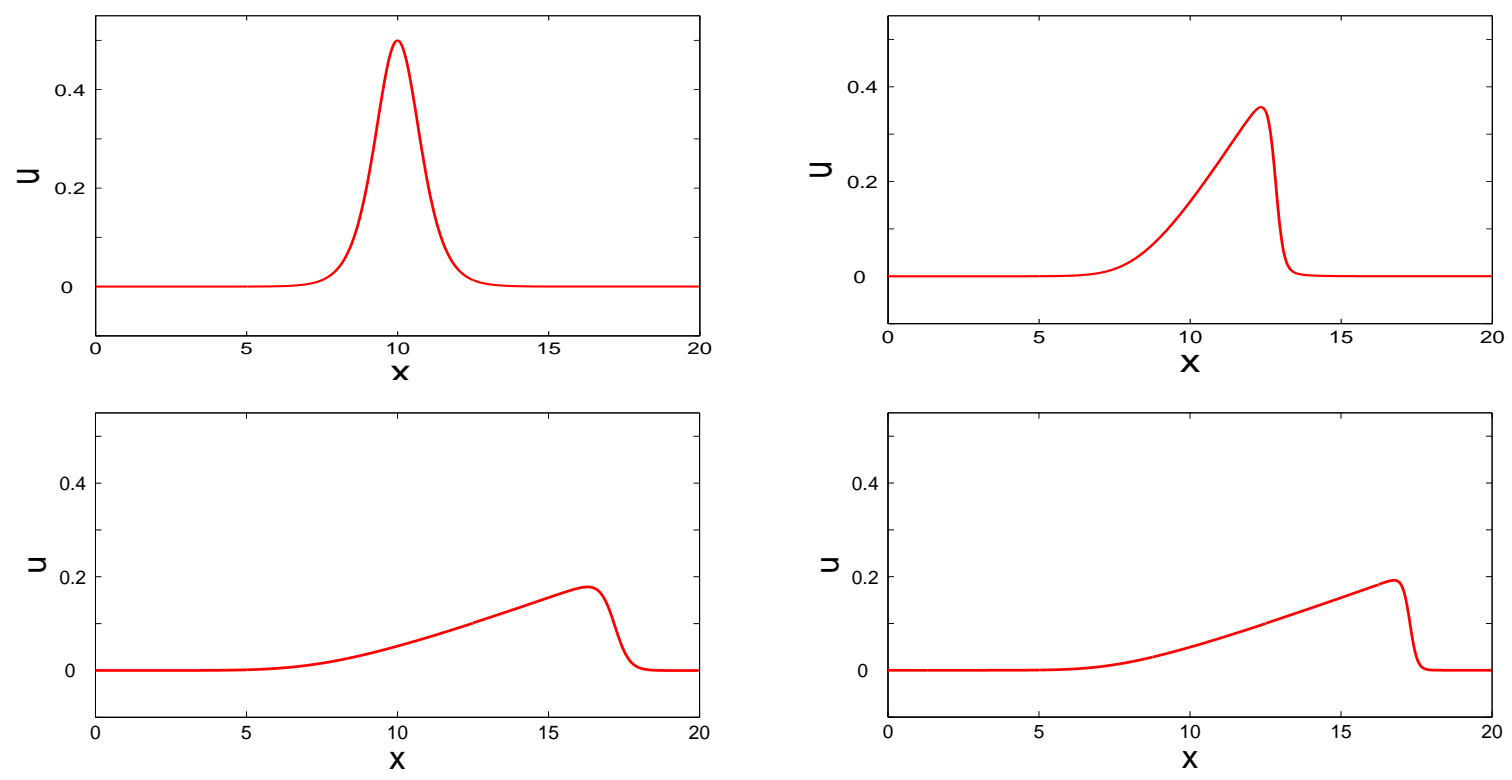

Figure 4: Simulation of the numerical scheme for the Burgers equation (6)-(8) with $a=2, b=0$, $d=1, z=3, y=4, s=1$ and $q=2.75$. We chose $\Delta x=0.025$. The equation parameters were $\mu=\Delta x=0.025$ and $c=1$ which implies $\Delta t=0.025$ and $\alpha^{2}=0.00073$. Upper left: initial condition $u(x, t=0)=0.5 \operatorname{sech}^{2}(x-L / 2)$ with $L=20$. Upper right: $u(x, t)$ at a later time $t=300$. Lower left: $u(x, t)$ at a later time $t=1600$. Lower right: as in the lower left figure but now with half the grid size $\Delta x=0.0125$ which implies $\alpha^{2}=0.00018$. During the time between two consecutive snapshots the wave has performed several revolutions across the periodic box.

chose $\Delta x=0.16$ and $\Delta t=0.12$ and used a standard semi-implicit Crank-Nicolson scheme with $a=b=1, d=0$ and $s=z=1$ and $y=0$. This particular scheme does not have the $\mathrm{KdV}$ equation as its modified equation up to $\mathcal{O}\left(\Delta x^{4}, \Delta t^{4}, \mu^{2} \Delta x^{2}, \mu^{2} \Delta t^{2}\right)$ since, for example, $y \neq z$. We employ again periodic boundary conditions. The initial condition disintegrates as expected for the KdV equation - in a train of solitary waves which subsequently elastically interact. Hence, a finite discretization $\Delta t$ and $\Delta x$ generates linear dispersion at leading order which balances the nonlinear steepening.

Our main concern in this paper however were special discretization schemes which use higherorder terms of the modified equations to achieve either more accurate behaviour of linearly dispersive regularization or a different type of regularization which supports approximations to shock solutions rather than solitary waves. We found discretization schemes such that the modified equation is the $\mathrm{KdV}$ equation up to $\mathcal{O}\left(\Delta x^{4}, \Delta t^{4}, \mu^{2} \Delta x^{2}, \mu^{2} \Delta t^{2}\right)$. We also found that for a particular choice of the discretization scheme the modified equation is the Camassa-Holm equation. Both equations support solitary waves. These waves are entirely artificial in the sense that the original system (1) supports shock waves rather than smooth solutions. The discretization associated with the $\mathrm{KdV}$ and the $\mathrm{CH}$ equation introduces linear and nonlinear dispersion. The linear numerical dispersion balances the nonlinear steepening giving rise to smooth solitary waves. Additionally, we derived numerical schemes whose modified equation is a proper regu- 


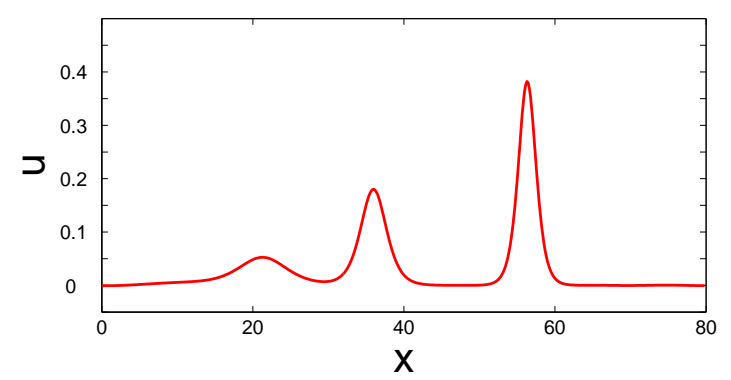

Figure 5: Snapshot of $u(x, t)$ at $t=2700$ for an arbitrary initial condition $u(x, t=0)=$ $0.25 \operatorname{sech}(0.2(x-L / 2))$ where $L=80$ is the box length. Equation parameters were $\mu=0.1$ and $c=1$. We used $\Delta t=0.1$.

larization of the Burgers equation in the sense that its solutions strongly converge to the weak shock solutions of the full Burgers equation when $\alpha^{2} \rightarrow 0$. However for the numerical scheme we proposed a small amount of viscosity was added to stabilize the scheme.

Crucial for our analysis is that the parent system already contains a dispersive term, i.e. the simple constant advection term $c u_{x}$ for the Burgers equation (1). For example, the Burgers equation without linear advection

$$
\frac{\partial u}{\partial t}+\mu u \frac{\partial u}{\partial x}=0
$$

would not allow for a resubstitution of temporal derivatives. From (21) we infer the modified equation for this equation to be

$$
\frac{\partial u}{\partial t}+\mu u \frac{\partial u}{\partial x}+\frac{1}{6} \mu z^{2} \Delta x^{2} u u_{x x x}+\frac{1}{2} \mu y^{2} \Delta x^{2} u_{x} u_{x x}=0 .
$$

The case $y=0$ was discussed in [14] where a semidiscretization of the Burgers equation was studied. It was shown that this "nonlinear" $\mathrm{KdV}$ equation behaves for small $\Delta x$ as the $\mathrm{KdV}$ equation.

Acknowledgements G.A.G gratefully acknowledges support by the Australian Research Council, DP0452147. G.A.G. is grateful for help with number theoretical questions to Claus Fieker and David Kohel.

\section{References}

[1] M. J. Ablowitz \& H. Segur, Solitons and the Inverse Scattering Transform SIAM: Philadelphia, (1981).

[2] H. S. Bhat and R. C. Fetecau, A Hamiltonian regularization of the Burgers equation, J. Nonlinear Sci. 16, 615-638 (2006).

[3] J. M. Burgers, A mathematical model illustrating the theory of turbulence, Adv. Appl. Mech. 1, 171-199 (1948). 
[4] H. S. Bhat, R. C. Fetecau, J. E. Marsden, K. Mohseni and M. West, Lagrangian averaging for compressible fluids, SIAM J. Multiscale Modeling and Simulation, 3, 818-837 (2005).

[5] H. S. Bhat and R. C. Fetecau, Lagrangian averaging for the 1d compressible Euler equation, Discrete and Continuous Dynamical Systems B, 6, 979-1000 (2006).

[6] R. Camassa \& D. D. Holm, An integrable shallow water equation with peaked solitons. Phys. Rev. Lett. 71, 1661-1664 (1993).

[7] A. Degasperis \& M. Procesi, Asymptotic integrability. In Symmetry and Perturbation Theory (ed. A. Degasperis \& G. Gaeta). World Scientific, 23-37 (1999).

[8] H. R. Dullin, G. A. Gottwald \& D. D. Holm, An integrable shallow water equation with linear and nonlinear dispersion, Phys. Rev. Lett. 87, 4501-4504 (2001).

[9] H. R. Dullin, G. A. Gottwald \& D. D. Holm, Camassa-Holm, Korteweg-de Vries-5 and other asymptotically equivalent equations for shallow water waves, Fluid Dynamics Research 33, 73-95 (2003).

[10] H. R. Dullin, G. A. Gottwald \& D. D. Holm, On asymptotically equivalent shallow water wave equations, Physica D 190, 1-14 (2004).

[11] D. R. Durran, Numerical methods for wave equations in geophysical fluid dynamics, New York: Springer, (1999).

[12] J. Frank, S. Reich, A. Staniforth, A. White \& N. Wood, Analysis of a regularized, timestaggered discretization method and its link to the semi-implicit method, Atmos. Sci. Let. 6, 97-104 (2005).

[13] U. Frisch, Turbulence: The legacy of A. N. Kolmogorov, Cambridge University Press, (1995)

[14] J. Goodman and P. D. Lax, On dispersive difference schemes. I, Comm. Pure Appl. Math. 41, 591-613 (1988).

[15] D. D. Holm, J. E. Marsden and T. S. Ratiu, Euler-Poincaré models of ideal fluids with nonlinear dispersion, Phys. Rev. Lett. 349 4173-4176 (1998).

[16] E. Infeld \& G. Rowlands, Nonlinear waves, solitons and chaos, Cambridge University Press, (2000).

[17] D. J. Korteweg \& G. de Vries, On the change of form of long waves advancing in a rectangular channel, and a new type of long stationary waves, Phil. Mag. (5) 39, 422-443 (1895).

[18] B. Leimkuhler \& S. Reich, Simulating Hamiltonian dynamics, Cambridge University Press, (2005).

[19] W. H. Press, S. A. Teukolsky, W. T. Vetterling \& B. P. Flannery, Numerical Recipes in C. Cambridge University Press (1992).

[20] T. Qian \& M. Tang, Peakons and periodic cusp waves in a generalized Camassa-Holm equation. Chaos, Solitons and Fractals 12, 1347-1360, (2001). 
[21] A. Scott, Nonlinear Science - Emergence and Dynamics of Coherent Structures (Oxford University Press, Oxford, 1999).

[22] G. B. Whitham, Linear and Nonlinear Waves, Wiley Interscience:New York, (1974). 